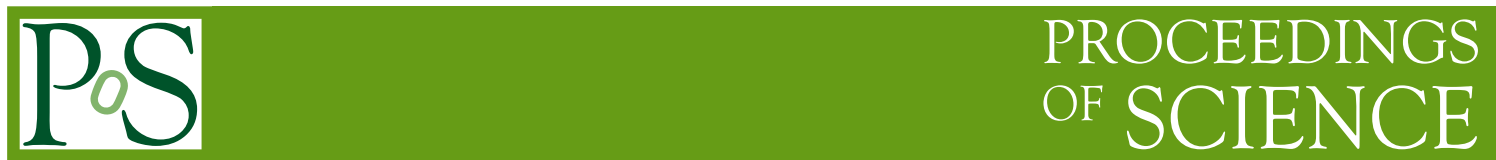

\title{
Searches for Hybrid Mesons at GlueX
}

\author{
Paul Mattione* \\ Carnegie Mellon University \\ E-mail: pmattejlab.org
}

The GlueX experiment at Jefferson Lab is designed to search for and study the spectrum of hybrid mesons, which are predicted to have explicit gluonic field contributions to their quantum numbers. The measurement of these spectra will provide access to the gluonic degrees of freedom of QCD in the confinement regime. A $12 \mathrm{GeV}$ electron beam incident on a diamond radiator will be used to produce a linearly-polarized, tagged-photon beam with a coherent peak at $9 \mathrm{GeV}$. The photon beam will be incident on a proton target located within the hermetic GlueX detector. The baseline design for the detector contains a start counter surrounding the target, a solenoidal magnet, a cylindrical central drift chamber, forward drift chamber planes, barrel and forward calorimeters, and a forward time-of-flight scintillator wall. In addition, a proposal has been submitted for adding a Cherenkov detector to facilitate identification of charged kaons. Many of the major detectors have been constructed and are being installed, with physics running scheduled to begin in 2016. The results of several simulated physics analyses are shown, demonstrating the ability of GlueX to measure the predicted spectrum of hybrid mesons.

$X V$ International Conference on Hadron Spectroscopy

$4-8 / 11 / 2013$

Nara, Japan

${ }^{*}$ Speaker. 


\section{Introduction}

Hybrid mesons, or mesons with excitations of their gluonic field, provide access to the gluonic degrees of freedom of QCD in the confinement regime. A rich spectrum of hybrid mesons has been predicted by lattice QCD [1], but there is contention over whether any of them have been unambiguously identified [2]. Some of the predicted hybrid mesons have an exotic combination of their total spin $(J)$, charge conjugation parity $(C)$, and intrinsic parity $(P)$ quantum numbers. These hybrid mesons are exotic because their $J^{P C}$ quantum numbers cannot arise from a valence $q \bar{q}$ pair alone. These exotic mesons, such as states with $0^{--}, 0^{+-}, 1^{-+}$, and $2^{+-}$quantum numbers, are easier to identify than their non-exotic counterparts because they cannot mix with non-hybrid $q \bar{q}$ states.

Jefferson Lab is in the midst of its $\sim \$ 300 \mathrm{M} 12 \mathrm{GeV}$ Upgrade, which is centered on doubling the maximum electron beam energy of the CEBAF accelerator to $12 \mathrm{GeV}$. A new experimental hall has been built to house the GlueX experiment, which is designed to map out the spectrum of hybrid mesons. It is scheduled to begin commissioning in late 2014, and to begin physics running in 2016. GlueX will take physics data for 60 days at $10^{7}$ tagged $\gamma / \mathrm{s}$ before ramping up to $5 \times 10^{7}$ tagged $\gamma / \mathrm{s}$ for 200 days.

\section{GlueX Experiment and Detector}

For the GlueX experiment, a $12 \mathrm{GeV}$ electron beam incident on a $20 \mu \mathrm{m}$ diamond radiator will be used to produce a linearly-polarized, coherent bremsstrahlung tagged-photon beam. The photon beam will be incident on a liquid hydrogen target located within the GlueX detector, shown in Figure 1. A photon beam will be used because exotic hybrid mesons are expected to be produced more easily from photon beams than pion beams due to angular momentum constraints. For a spin0 pion to couple to exotic mesons, one of its quark spins must flip, whereas a spin-1 photon can couple by fluctuating into a virtual $q \bar{q}$ system. The polarization of the photon beam will be used to facilitate a more precise identification of the hybrid meson states and their quantum numbers.

The tagged-photon beam will have a linear polarization of $\sim 40 \%$ at its $9 \mathrm{GeV}$ coherent peak. A fixed-counter array will be used to tag the photon beam energy from $3.0 \mathrm{GeV}$ to $11.8 \mathrm{GeV}$ with varying resolution. An adjustable microscope will be used to tag the photon beam energy in the $8.4 \mathrm{GeV}$ to $9.0 \mathrm{GeV}$ coherent peak at a resolution of $0.1 \%$. These detectors are currently under construction, and will be installed in Spring 2014. The diamond radiators will be fabricated in Fall 2014.

The central drift chamber (CDC), shown in Figure 2a, is $1.5 \mathrm{~m}$ long and contains 28 rings of straw tubes [3]. Sixteen of the rings have been rotated by $\pm 6^{\circ}$ to provide $\theta$ and $z$ information. The forward drift chambers (FDC), show in Figure 2b, consist of four detector packages. Each package contains six planes of anode wires, rotated $60^{\circ}$ with respect to each other, and cathode strips rotated by $\pm 75^{\circ}$. The CDC and FDC provide overall position resolutions of about $150 \mu \mathrm{m}$ and $200 \mu \mathrm{m}$, respectively. The reconstructed momentum resolution $\sigma_{p} / p$ is about $1 \%$ to $3 \%$ for tracks at $\theta>5^{\circ}$. Together, these drift chambers enable charged track reconstruction from $1^{\circ}$ to $155^{\circ}$. Both the $\mathrm{CDC}$ and FDC packages have been constructed, and are scheduled for installation in early 2014. 


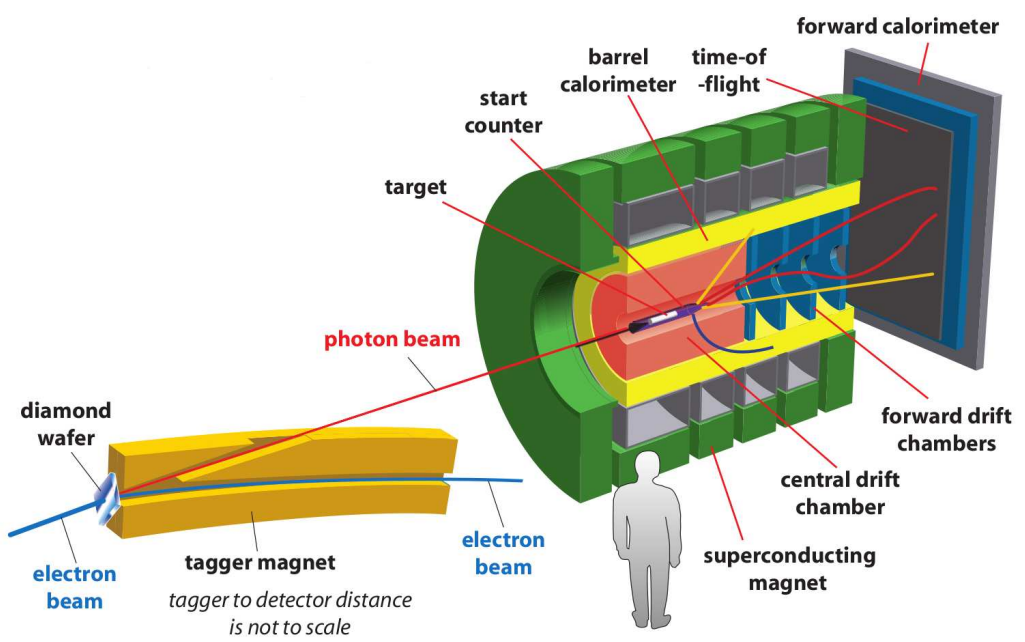

Figure 1: A schematic of the GlueX detector. See text for details.

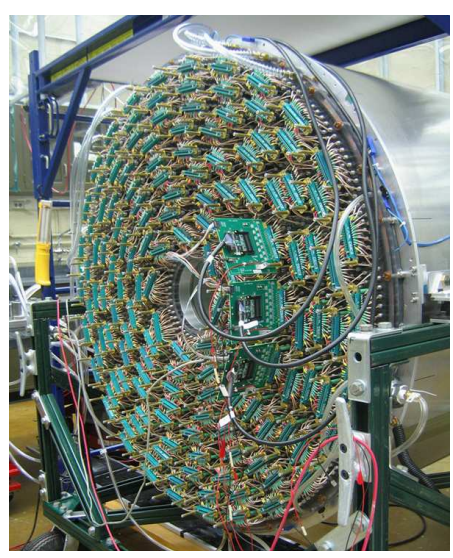

(a) GlueX central drift chamber.

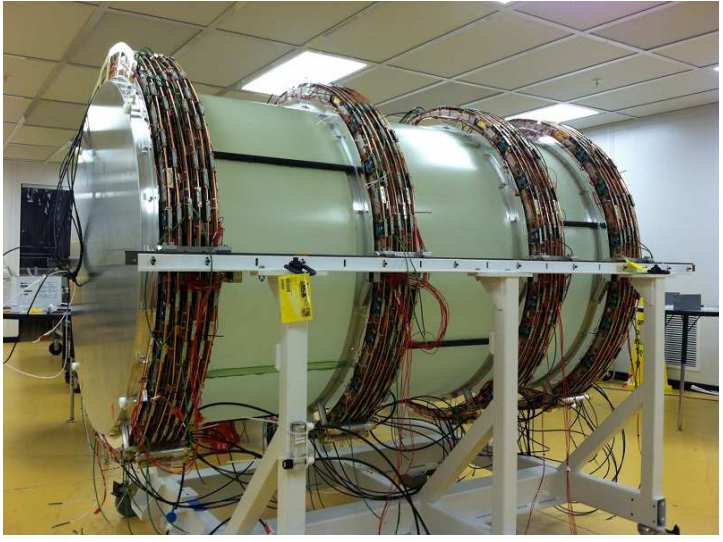

(b) GlueX forward drift chamber assembly.

Figure 2: The GlueX central and forward drift chamber systems. See text for details.

The barrel calorimeter (BCAL), shown in Figure 3a, is composed of 48 4-m-long modules. These modules contain scintillating fibers embedded in a lead matrix that is 15.1 radiation lengths thick. The forward calorimeter (FCAL), shown in Figure 3b, contains 2800 lead-glass blocks. The energy resolutions $\sigma_{E} / E$ of the BCAL and FCAL are 5.5\%/ $\sqrt{E}+2.0 \%$ and $5.6 \% / \sqrt{E}+3.5 \%$, respectively [4]. Together, the calorimeters enable neutral particle reconstruction from $2^{\circ}$ to $120^{\circ}$. Both detectors have been constructed and installed in the experimental hall.

A combination of several detector systems will be used to perform particle identification. The start counter will be used to identify the RF beam bunch associated with each event. It consists of 30 thin scintillator paddles, and will have a resolution of about 350 ps. Construction of the start counter will finish in Spring 2014, and it will be installed in Summer 2014. The forward time-offlight detector consists of two planes of scintillator paddles at $90^{\circ}$ to each other. The system has a timing resolution of $70 \mathrm{ps}$, providing $>4 \sigma \mathrm{kaon} /$ pion separation at momenta up to $2 \mathrm{GeV} / \mathrm{c}$. It has an angular coverage from $2^{\circ}$ to $11^{\circ}$, and will finish being installed in early 2014 . A proposal 


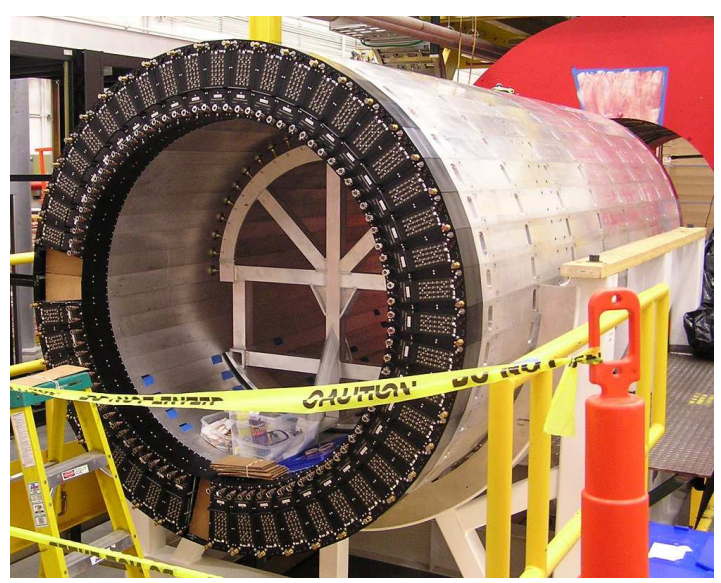

(a) GlueX barrel calorimeter.

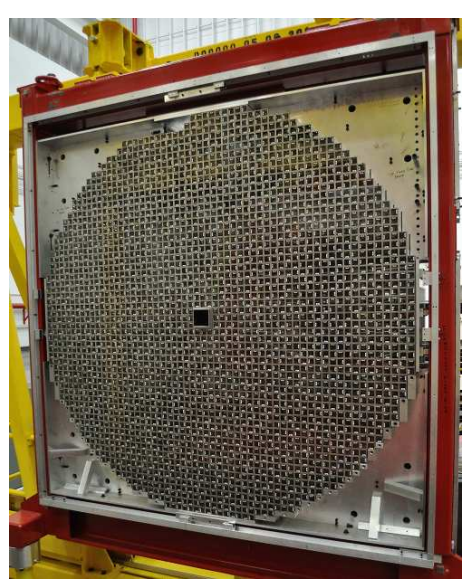

(b) GlueX forward calorimeter.

Figure 3: The GlueX barrel and forward calorimeters. See text for details.

\begin{tabular}{|c|c|c|c|c|c|c|c|}
\hline Channel & $\pi^{+} \pi^{+} \pi^{-}$ & $\pi^{+} \pi^{-} \pi^{0}$ & $\omega_{3 \pi} \pi \pi$ & $K K \pi \pi$ & $\eta_{\gamma \gamma} \pi \pi$ & $K K \pi$ & $\eta_{\eta \pi \pi}^{\prime} \pi$ \\
\hline Cross Section $(\mu \mathrm{b})$ & 10.0 & 2.0 & 0.2 & 0.5 & 0.2 & 0.1 & 0.1 \\
\hline Signal Yield $\left[10^{6}\right]$ & 3000 & 600 & 40 & 40 & 30 & 10 & 3 \\
\hline
\end{tabular}

Table 1: Predicted cross sections and estimated experimental yields for several channels [6].

for a focusing DIRC detector has been submitted, which would use 48 of the Babar DIRC bars. This design would provide $>3 \sigma \mathrm{kaon} /$ pion separation at momenta up to $4 \mathrm{GeV} / \mathrm{c}$, which would significantly help in improving the mapping of the spectrum of strange mesons. Finally, timing information from the BCAL and $d E / d x$ in the drift chambers will be used to identify slower, large angle tracks.

\section{Simulated Analyses}

Several studies have been performed to test the capability of GlueX to study the spectrum of hybrid mesons. Table 1 shows the expected experimental yields for several channels to which hybrid mesons are predicted to decay [6]. These yields were determined using approximations for detector efficiencies, and are quoted for the predicted cross sections shown in the table. In addition, the amplitude analysis shown in Figure 4 demonstrates the extraction of a simulated, percent-level amplitude for the $\pi_{1}(1600)$ hybrid meson from a sample of pure $\pi^{+} \pi^{+} \pi^{-} n$ events [6].

In addition, several background subtraction studies have been performed using a PYTHIAbased event generator. Boosted decision trees (BDTs) [5] were used to select events for each topology. A BDT is a type of multivariate classifier that uses machine learning to train on simulated measurements from separate signal and background events to optimize signal/background separation of experimental data. For $\gamma p \rightarrow \pi^{+} \pi^{+} \pi^{-} n$, GlueX can measure signal events with $22 \%$ efficiency at $95 \%$ signal purity. Table 2 shows the ability of GlueX to extract various strangeness final states without the proposed Cherenkov detector [6]. These efficiencies do not include acceptance effects. 

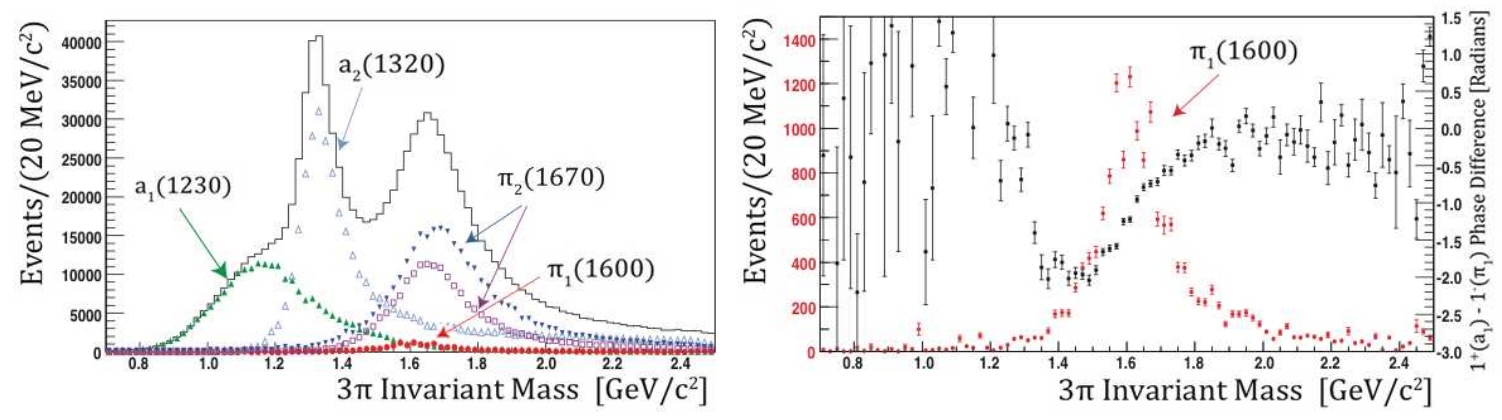

Figure 4: Amplitude analysis for extraction of a $\pi_{1}(1600)$ resonance simulated at $1.6 \%$. The left plot shows the resonant components in each mass bin. The right plot shows the $\pi_{1}(1600)$ resonance amplitude in red, and the phase difference between the $\pi_{1}(1600)$ and $a_{1}(1230)$ in black [6].

\begin{tabular}{|c|c|c|c|}
\hline Resonance Decay & Final State & Selection Efficiency & Signal Yield $\left[10^{6}\right]$ \\
\hline \hline$\phi_{3}(1850) \rightarrow K^{+} K^{-}$ & $K^{+} K^{-}$ & 0.73 & 1.5 \\
\hline$Y(2175) \rightarrow \phi f_{0}(980)$ & $K^{+} K^{-} \pi^{+} \pi^{-}$ & 0.53 & 0.46 \\
\hline$\eta_{1}^{\prime}(2300) \rightarrow K^{*} K_{S}$ & $K^{+} \pi^{-} \pi^{+} \pi^{-}$ & 0.32 & 5.3 \\
\hline$h_{2}^{\prime}(2600) \rightarrow K_{1}^{+} K^{-}$ & $K^{+} K^{-} \pi^{+} \pi^{-}$ & 0.26 & 0.12 \\
\hline$\Xi^{-}(1820) \rightarrow \Lambda K^{-}$ & $p \pi^{-} K^{-}$ & 0.36 & 0.09 \\
\hline
\end{tabular}

Table 2: Estimated selection efficiency and yields for several channels at 90\% signal purity [6].

\section{Conclusion}

Mapping the spectrum of hybrid mesons is critical for determining the gluonic degrees of freedom of QCD. The GlueX experiment is ideally suited for this study, and its detectors are currently being constructed and installed. The experiment is scheduled to begin physics running in 2016 .

\section{Acknowledgments}

This work was supported in part by U.S. Department of Energy Grant No. DE-FG02-87ER40315.

\section{References}

[1] J. Dudek, Phys. Rev. D 84, 074023 (2011).

[2] C. A. Meyer and Y. Van Haarlem, Phys. Rev. C 82, 025208 (2010).

[3] Y. Van Haarlem et al., Nucl. Instrum. Meth. A 622, 142 (2010).

[4] K. Moriya et al., Nucl. Instrum. Meth. A 726, 60 (2013).

[5] L. Brieman et al., Classification and regression trees, Wadsworth International Group, Belmont, California (1984).

[6] A. AlekSejevs et al. (The GlueX Collaboration), Jefferson Lab PAC 40 Proposal, PR12-13-003 (2013) [arXiv:1305.1523 [nucl-ex]]. 